\title{
EFFECTS OF THE DEVIATION ANGLE OF THE BOREHOLE IN THE INDUCTION ANISOTROPY LOGS
}

\author{
Paulo Roberto de Carvalho ${ }^{1}$, Cícero Roberto Teixeira Régis ${ }^{2}$ and Valdelírio da Silva e Silva ${ }^{3}$
}

\begin{abstract}
This paper performs an analysis of the effects of the well's deviated angle on the tensor triaxial induction tool signals within a thinly sand-shale laminated reservoirs and their equivalent intrinsic anisotropic models. The responses from coaxial and coplanar coil arrays in inclined wells are studied in detail, including the analysis of their apparent anisotropy logs, as well as their estimation of sand conductivity in the environments with a structural anisotropy. The dip angle effects are modeled in simple geometries as one-dimensional (1D) models, neglecting the presence of the borehole and the invasion zones, since they provide basic insight for understanding tool responses in more complex models. The results show a strong sensitivity of both the coaxial and coplanar signals to the deviated angle. It is verified that the anisotropy values are significantly reduced when the well is inclined as compared to what is found for the true vertical case, even for inclinations small enough for the wells to be classified as technically vertical (30 degrees or less). Therefore, the angle effects must be carefully considered, even for technically vertical wells. Otherwise, potential finely laminated reservoirs can be underestimated or even ignored.
\end{abstract}

Keywords: deviated well logging, tensor induction tool, laminated reservoirs, electrical anisotropy.

RESUMO. Neste artigo é feita uma análise dos efeitos do ângulo de inclinação dos poços nos sinais da ferramenta de indução triaxial dentro de reservatórios finamente laminados e seus respectivos modelos anisotrópicos equivalentes. Aqui são mostradas apenas as respostas dos pares de bobinas coaxial e coplanar, que são os utilizados para obter os perfis de anisotropia aparente, bem como para estimar a condutividade das lâminas de areia nesses ambientes de anisotropia estrutural. Os efeitos do ângulo de inclinação são modelados em geometrias relativamente simples como, por exemplo, modelos unidimensionais (1D), negligenciando a presença do poço e das zonas de invasão, uma vez que estas simulações fornecem informações básicas para compreender as respostas da ferramenta em modelos mais complexos. Os resultados mostram uma forte sensibilidade dos sinais dos arranjos coaxiais e coplanares ao ângulo de inclinação do poço, refletindo numa significativa redução dos valores de anisotropia aparente. Assim, mostramos que é imperativo monitorar estes efeitos do ângulo mesmo em situações consideradas tecnicamente verticais (30 graus ou menos). Caso contrário, potenciais reservatórios de camadas finas podem ser subestimados ou até mesmo ignorados.

Palavras-chave: poços desviados, ferramentas triaxiais, reservatórios laminados, anisotropia elétrica.

\footnotetext{
${ }^{1}$ Universidade Federal Rural da Amazônia, Instituto Ciberespacial, Av. Presidente Tancredo Neves, 2501, Terra Firme, 66077-530 Belém, PA, Brazil. Phone: +55(91) 3210-5109 - E-mails: paulo.carvalho@ufra.edu.br; prdcarvalhoufra@gmail.com

2 Universidade Federal do Pará, Faculdade de Geofísica, Rua Augusto Corrêa, 01, 66075-110 Belém, PA, Brazil. Phone: +55(91) 3201-7692; and National Institute of Science and Technology of Petroleum Geophysics, Universidade Federal da Bahia, Instituto de Geociências, sala 312-C, Campus de Ondina, 40170-115 Salvador, BA, Brazil-E-mails: cicero@ufpa.br; ciceroregis@gmail.com

${ }^{3}$ Universidade Federal do Pará, Campus Castanhal, Faculdade de Matemática. Av. dos Universitários, s/n, Jaderlândia, 68746-630 Castanhal, PA, Brazil. Phone: +55(91) 3311-4608 - E-mails: valdel@ufpa.br; valdeliriosilva@gmail.com
} 


\section{INTRODUCTION}

Current triaxial, multicomponent or tensor induction probes consist basically of a combination of a coaxial arrangement with two coplanar arrangements of coils (Fig.1-a), i.e., three sources and three sensors, with axes orthogonal to each other (Kriegshauser et al., 2000). These probes were designed originally to investigate thinly laminated formations with a structural anisotropic behavior in vertical wells. The responses of the various arrangements of coils are simultaneously registered on multiple channels at multiple frequencies and source-sensor spacing allowing the interpreter to characterize the anisotropic properties of these important geological formations for oil and gas.

Currently, besides being the main location tool of finely laminated reservoirs, these sets of sources and sensors are also applied in many situations of asymmetric geometry, such as locating dissolution cavities (vugs) and fractured zones in the vicinity of the wells, monitoring invasion fronts in horizontal wells, among others (Omeragic et al., 2015).

In this work, we show some numerical responses for one-dimensional (1D) layered anisotropic geoelectric models, in which the presence of the borehole and the invasion zones are neglected, to simulate deviated logs with respect to bedding within geological environments of hydrocarbon reservoirs with structural electrical anisotropy.

Our goals here are to investigate the effects of the dip angle in the induction tool response and to investigate quantitatively how much the probe's inclination affects the apparent anisotropy logs obtained from the ratio of the coaxial to the coplanar responses.

\section{THEORY AND ANALYSIS METHOD}

In the theoretical treatment, the coils are represented as point magnetic dipoles, as illustrated in Figure 1-b. The magnetic field components from the vertical (VMD) and horizontal (HMD) magnetic dipoles are calculated as described in Carvalho et al. (2010) for an isotropic multilayered medium without the borehole and invasion zones. The magnetic field components from the HMD within an anisotropic medium are calculated as described in Kaufman \& Itskovich (2017). The semi-analytical responses of these $1 \mathrm{D}$ models result in improper integrals due to the inverse Hankel transform, which are solved numerically with a 21-point Gauss-Kronrod quadrature rule.

The fields from an arbitrary inclined dipole are calculated as a combination of those from a vertical $\left(H^{v}\right)$ and a horizontal $\left(H^{h}\right)$ source. Equations (1) and (2) yield the coaxial $\left(H^{c x}\right)$ and coplanar $\left(H^{c p}\right)$ responses in terms of the component of the magnetic field normal to their receivers. The dip angle $\theta$ in Figure 2 is determined by the orientation of the borehole (azimuth and deviation) and the orientation of the formation (dip and strike). This angle can be caused by deviated wells in flat formations, by vertical wells in dipping beds, or by any combination thereof.

$$
\begin{aligned}
H^{c x} & =\left(H_{z}^{v}+H_{z}^{h}\right) \cos \theta+\left(H_{x}^{v}+H_{x}^{h}\right) \sin \theta, \\
H^{c p} & =\left(H_{z}^{v}+H_{z}^{h}\right) \sin \theta+\left(H_{x}^{v}+H_{x}^{h}\right) \cos \theta .
\end{aligned}
$$

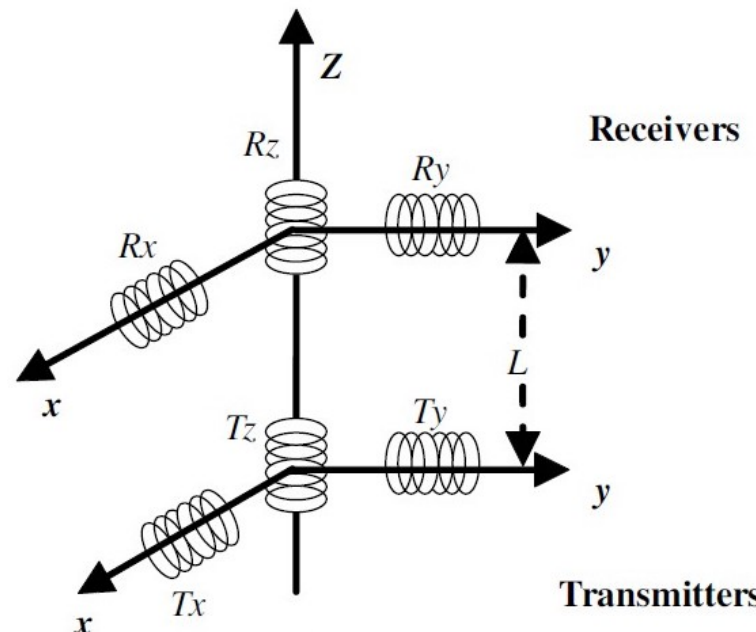

(a)

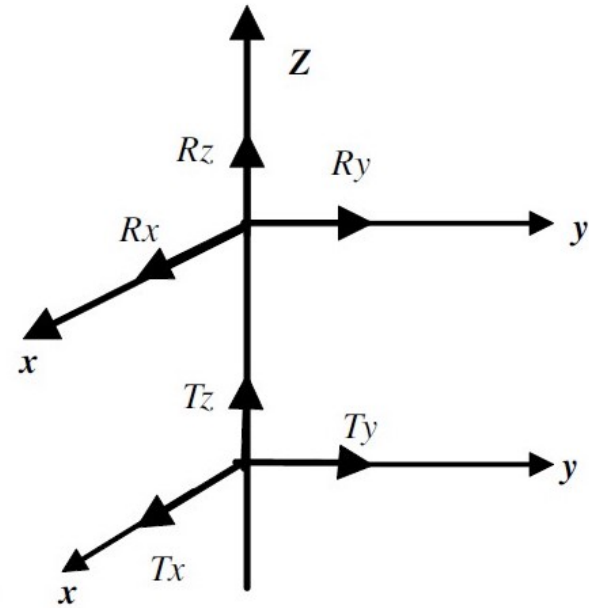

(b)

Figure 1 - (a) Basic structure of the multicomponent induction tool and (b) its equivalent model of magnetic dipoles (Zhang et al., 2012). 


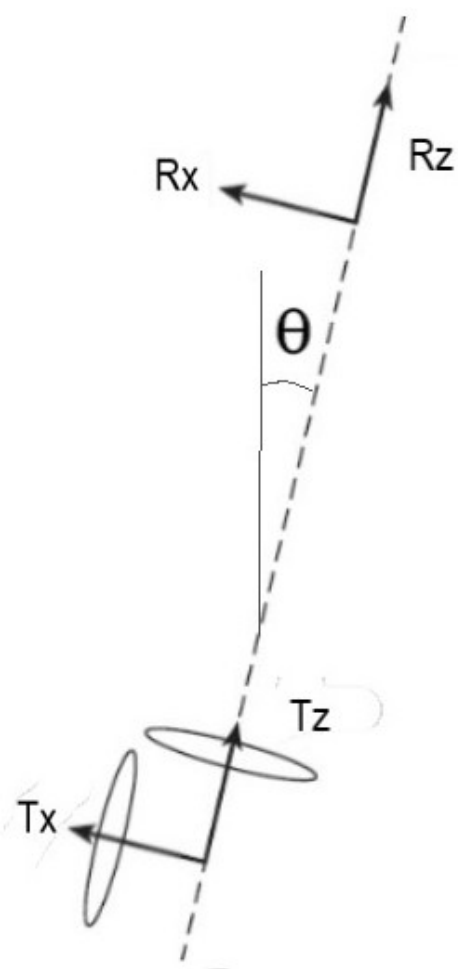

Figure 2 - The coaxial ( $T z-R z)$ and coplanar $(T x-R x)$ dipole model within a deviated well (adapted from Ellis \& Singer (2007)).

The complex conductivity may be written in terms of the magnetic fields registered at each receiver coil as

$$
\begin{aligned}
\sigma_{R}^{c x}+i \sigma_{X}^{c x} & =\left(\frac{2 i}{\omega \mu L^{2}}\right) h^{c x}, \\
\sigma_{R}^{c p}+i \sigma_{X}^{c p} & =\left(\frac{-2 i}{\omega \mu L^{2}}\right) h^{c p},
\end{aligned}
$$

where $h^{c x}$ and $h^{c p}$ are the secondary magnetic fields, which come exclusively from the medium, i.e., without the transmitter/receiver mutual coupling terms, and $L$ is the coil spacing.

The coaxial (Moran \& Kunz, 1962) and coplanar (Carvalho \& Verma, 1999) boosted signals with a partial Skin Effect Correction (S.E.C) are obtained by

$$
\begin{aligned}
\sigma_{c}^{c x} & =\frac{\sigma_{R}^{c x}}{1-(2 / 3)\left(L / \delta^{c x}\right)}, \\
\sigma_{c}^{c p} & =\frac{\sigma_{R}^{c p}}{1-(4 / 3)\left(L / \delta^{c p}\right)},
\end{aligned}
$$

where $\delta^{c x}=\sqrt{2 /\left(\omega \mu \sigma_{R}^{c x}\right)}$ and $\delta^{c p}=\sqrt{2 /\left(\omega \mu \sigma_{R}^{c p}\right)}$.

When source and receiver coil axes are both oriented perpendicular to the horizontal bedding planes, as in the case of the vertical coaxial array $\left(\boldsymbol{\theta}=0^{\circ}\right)$, only the component of the conductivity parallel to the planes (horizontal conductivity $\sigma_{h}$ ) affects the response. On the other hand, when both coils have axes oriented parallel to the bedding planes, as in the case of a vertical coplanar array, the vertical conductivity $\sigma_{v}$ also affects the response significantly.

The vertical coaxial signal will be biased towards the high conductivity laminations (no oil-shale) because the circular induced currents flow parallel to the bedding planes, which implies that the anisotropy has no effect. However, elliptical induced currents from a vertical coplanar array will cross the bed interfaces, so that polarization charges accumulate at these boundaries. The coplanar signal is due to both horizontal $\left(\sigma_{h}\right)$ and vertical $\left(\sigma_{v}\right)$ conductivities, with $\sigma_{h}$ usually larger than $\sigma_{v}$ in clastic sedimentary formations.

In Transversely Isotropic layers with a Vertical axis of symmetry (TIV), in which the main anisotropy directions are the same as the coordinate axes, the conductivity tensor reduces to

$$
\sigma=\left[\begin{array}{ccc}
\sigma_{h} & 0 & 0 \\
0 & \sigma_{h} & 0 \\
0 & 0 & \sigma_{v}
\end{array}\right]
$$

For this type of anisotropic medium, a characteristic parameter named coefficient of electrical anisotropy is defined as

$$
\lambda^{2}=\frac{\sigma_{h}}{\sigma_{v}} .
$$

Kaufman \& Itskovich (2017) deduced through current density distribution and Anderson et al. (2008) show through circuit theory (parallel and series resistors) the same relation between the horizontal and vertical conductivities of the homogeneous anisotropic media and the two conductivities of the thinly laminated medium as, for example, formed by two alternating sand-shale laminae $\left(\sigma_{s d}\right.$ and $\left.\sigma_{s h}\right)$ when their thicknesses are less than the tool's vertical resolution:

$$
\begin{aligned}
& \sigma_{h}=\sigma_{s d} V_{s d}+\sigma_{s h} V_{s h} \\
& \sigma_{v}=\left(\frac{V_{s d}}{\sigma_{s d}}+\frac{V_{s h}}{\sigma_{s h}}\right)^{-1},
\end{aligned}
$$

where $V_{s d}$ and $V_{s h}$ are the volume fractions of each material which are obtained by spectroscopy probe. Thus, the sand laminae conductivities $\sigma_{s d}$ can be estimated from the horizontal $\left(\sigma_{h} \approx\right.$ $\sigma_{c}^{c x}$ ) and vertical $\left(\sigma_{v} \approx \sigma_{c}^{c p}\right)$ conductivities (Eqs. 9 and 10 ) and, finally, their reciprocal values may be applied in Archie's equation to estimate the water saturation in the thinly laminated reservoir (Clavaud et al., 2005). 

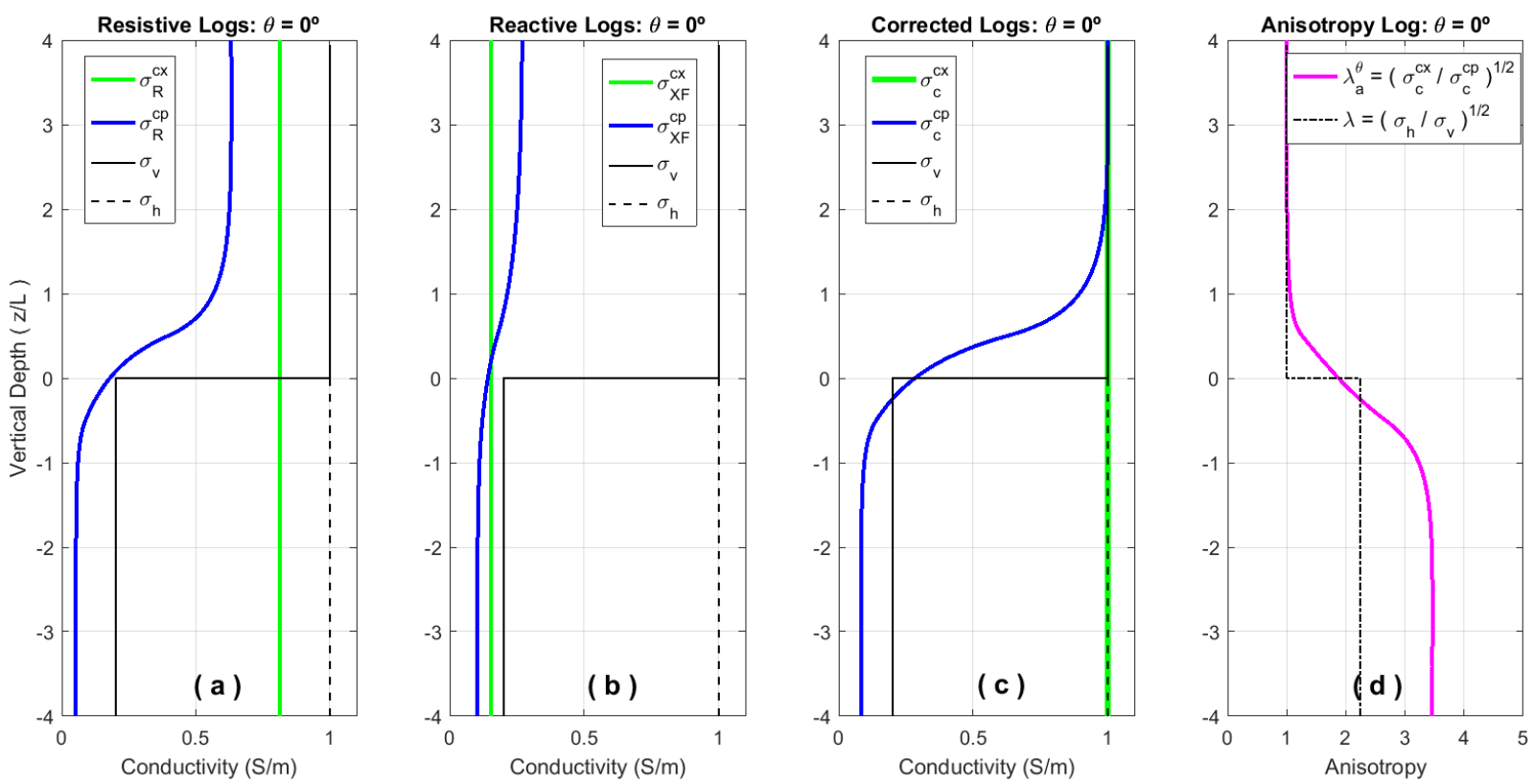

Figure 3 - Resistive (a), reactive (b), corrected (c) and anisotropy (d) vertical logs of the coaxial and coplanar coil arrays for the two half-space anisotropic model presented in Zhdanov et al. (2001).
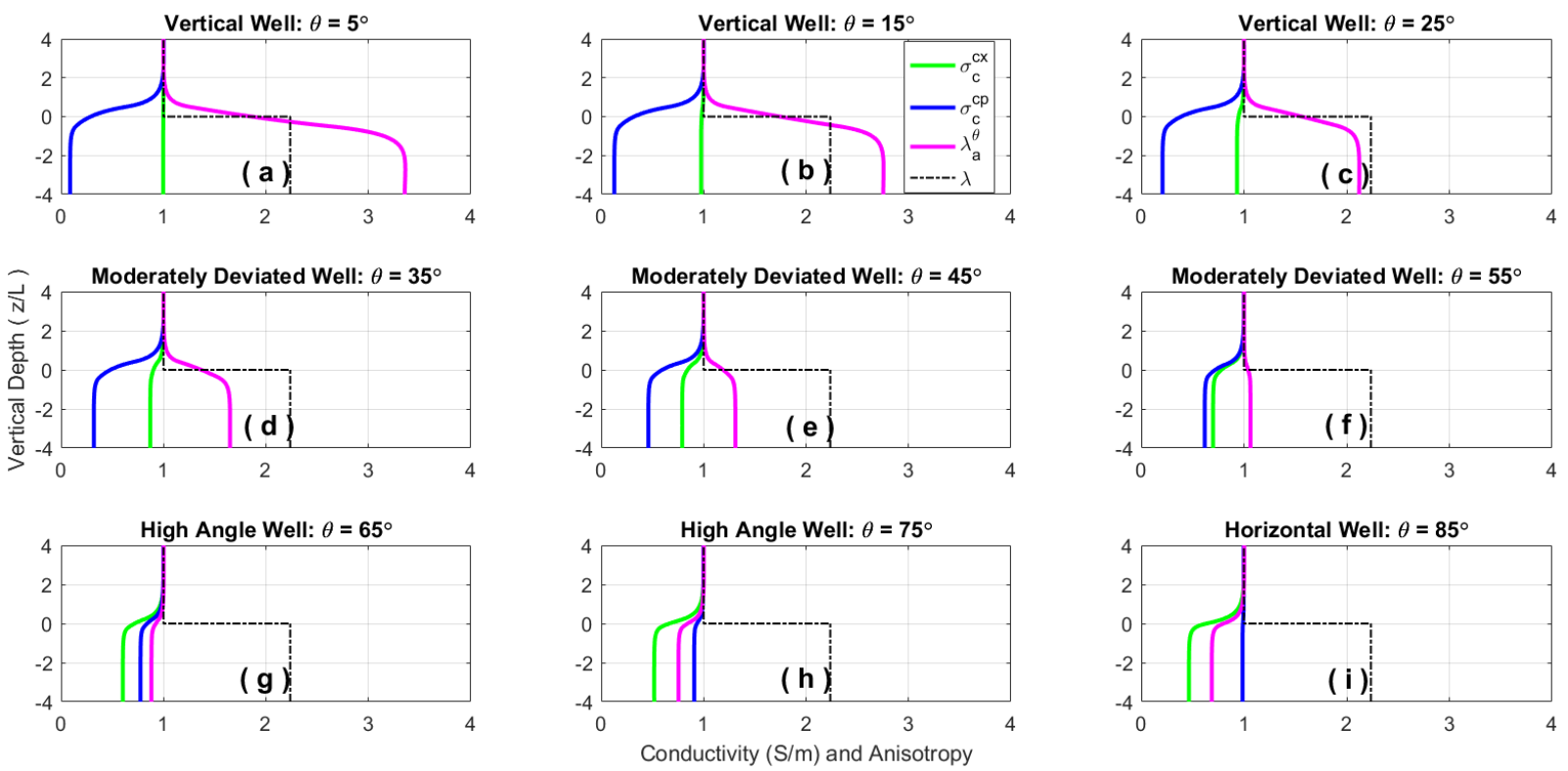

Figure 4 - Effect of the deviated angle on the coaxial and coplanar corrected logs and on the resulting anisotropy logs for a two half-space anisotropic model.

Kaufman \& Itskovich (2017) show that in the low frequency range $(L / \delta \ll 1)$ the quadrature component of the secondary magnetic field (without the mutual term) registered by vertical coplanar array is directly proportional to the vertical conductivity $\left(\sigma_{v}\right)$. For this reason, measuring the ratio of the quadrature components of the coaxial $Q\left\{h_{z}^{c x}\right\}$ and coplanar $Q\left\{h_{x}^{c p}\right\}$ arrays allows us to obtain an apparent coefficient of anisotropy $\left(\lambda_{a}^{2}\right)$ or simply electrical anisotropy index which is also given by the ratio of the corrected signals (S.E.C.) of the coplanar and coaxial arrays:

$$
\lambda_{a}^{2}=\frac{Q\left\{h_{x}^{c p}\right\}}{Q\left\{h_{z}^{c x}\right\}}=\frac{\sigma_{c}^{c p}}{\sigma_{c}^{c x}}
$$


Anderson et al. (2008) show that this anisotropic index is a useful measurement for determining the level of anisotropy, and that when this ratio is higher than five, it alerts the log analyst to look for potential laminated pay-reservoir.

\section{RESULTS AND DISCUSSION}

We believe that analyzing the angle effect in simple geometries provides basic insight for understanding tool responses in more complex models.

The vertical coordinates in some figures defined as "vertical depth" is the distance from any one point in the deviated well to the horizontal bed boundaries models and it is obtained from the depth measured by logs along the borehole.

Figure 3 shows, from left to right, the resistive (a), reactive (b), corrected (c) and anisotropy (d) logs for the vertical coaxial and coplanar arrays in a two half-space anisotropic model presented in Zhdanov et al. (2001), simulating an interface of the two very thick anisotropic beds. The black straight lines show the true values of the parameters of this model. The horizontal conductivities are the same $(1.0 \mathrm{~S} / \mathrm{m})$ in both layers, that is, there is no horizontal conductivity contrast. The vertical conductivities of the top and bottom beds are different: $1.0 \mathrm{~S} / \mathrm{m}$ and $0.2 \mathrm{~S} / \mathrm{m}$, respectively, a vertical conductivity contrast of 5 times. Thus, only the bottom bed is a truly anisotropic medium while the top bed behaves as an isotropic medium.

The remarkable result is that all the coaxial logs (green lines) are rectilinear because this coil array is totally blind to the vertical conductivity and, consequently, ignores the bed interface. On the other hand, all coplanar logs (blue lines) show deflections indicating clearly the existence of these two different media. As we have already described in Equation 11, the anisotropy log of track (d) is obtained through the ratio between the coaxial and coplanar corrected logs (track c) and, consequently, it reflects the sensitivity of the coplanar signal to the anisotropy. According to Zhdanov et al. (2001) this anisotropic model is a very good example of a practical situation where conventional induction logging (coaxial arrays) can miss a geological structure.

According to Passey et al. (2005) the industry standard to define a vertical well is one in which the apparent deviation angle with respect to bedding is less than $30^{\circ}$; a moderately deviated well as one where the apparent deviation angle is between $30^{\circ}$ and $60^{\circ}$; a high angle well as one where the angle is between $60^{\circ}$ and $80^{\circ}$; and a horizontal well as one where the angle is greater than $80^{\circ}$.
Figure 4 shows the angle effect of the deviated wells on the coaxial and coplanar corrected logs and in their resulting anisotropy logs. The deflections in the coplanar logs and, consequently, in the apparent anisotropy logs register the presence of the two different media. As the dip angle ranges from $5^{\circ}$ to $85^{\circ}$, these deflections progressively decrease, so that between $55^{\circ}$ and $65^{\circ}$ the anisotropy mean value is close to one (isotropic medium) and at $85^{\circ}$ the coplanar log becomes rectilinear. However, the coaxial logs show an opposite behavior, that is, as the angle increases, they begin to show a sensitivity to the vertical conductivity and, consequently, a deflection emerges and grows to match (between $55^{\circ}$ and $65^{\circ}$ ) and then overcome the deflections in the coplanar logs at the highest angles.

Figure 5 shows the absolute (a) and relative (b) effects of the dip angle within the bottom anisotropic medium, where the apparent anisotropic values are taken at $z=-3 L$, far enough below the bed interface that its effect is negligible and the measurements are influenced only by the anisotropic medium. As the deviation angle ranges from $0^{\circ}$ to $85^{\circ}$, the anisotropy signal $\lambda_{a}^{\theta}$ decreases from the value of $\lambda^{\nu}$. From $0^{\circ}$ to $30^{\circ}$ the apparent anisotropy drops approximately $45 \%$, although these well deviations are still considered technically vertical.

Figure 6 shows a model with a structural anisotropy (TIV) created by the alternation of conductive (shale) and resistive (hydrocarbon-bearing sand) laminae $(h=L / 2)$. This structure is closer to actual geological situations than the intrinsically anisotropic medium shown in Figure 3. The coaxial (a) and coplanar (b) coil arrays traverse vertically $\left(\boldsymbol{\theta}=0^{\circ}\right)$ a thick package $(H=10.5 L)$ of a laminated formation with symmetrical shoulders. There is a relatively low conductivity contrast between the sand-shale isotropic laminae with conductivities $\sigma_{s d}=0.2$ $\mathrm{S} / \mathrm{m}$ and $\sigma_{s h}=1.0 \mathrm{~S} / \mathrm{m}$, and their volume fractions are the same $\left(V_{s d}=V_{s h}=0.5\right)$.

The coplanar signals (Fig. 6-b) show a more prominent oscillation within the package, and they suffer the strongest adjacent bed and skin effects. They also show a greater sensitivity to the resistive laminae, whereas the coaxial signals (Fig. 6-a) suffer a stronger effect of the conductive media, which masks the presence of the resistive laminae.

Polarization "horns" appear in the coplanar logs against the package boundaries, which are more evident on the resistive signal. These horns are caused by surface charge build-up at the boundaries, since the normal component of the electric field is discontinuous at the interfaces. However, polarization horns do not appear in the coplanar logs of the Figures 3 and 4 in which the 

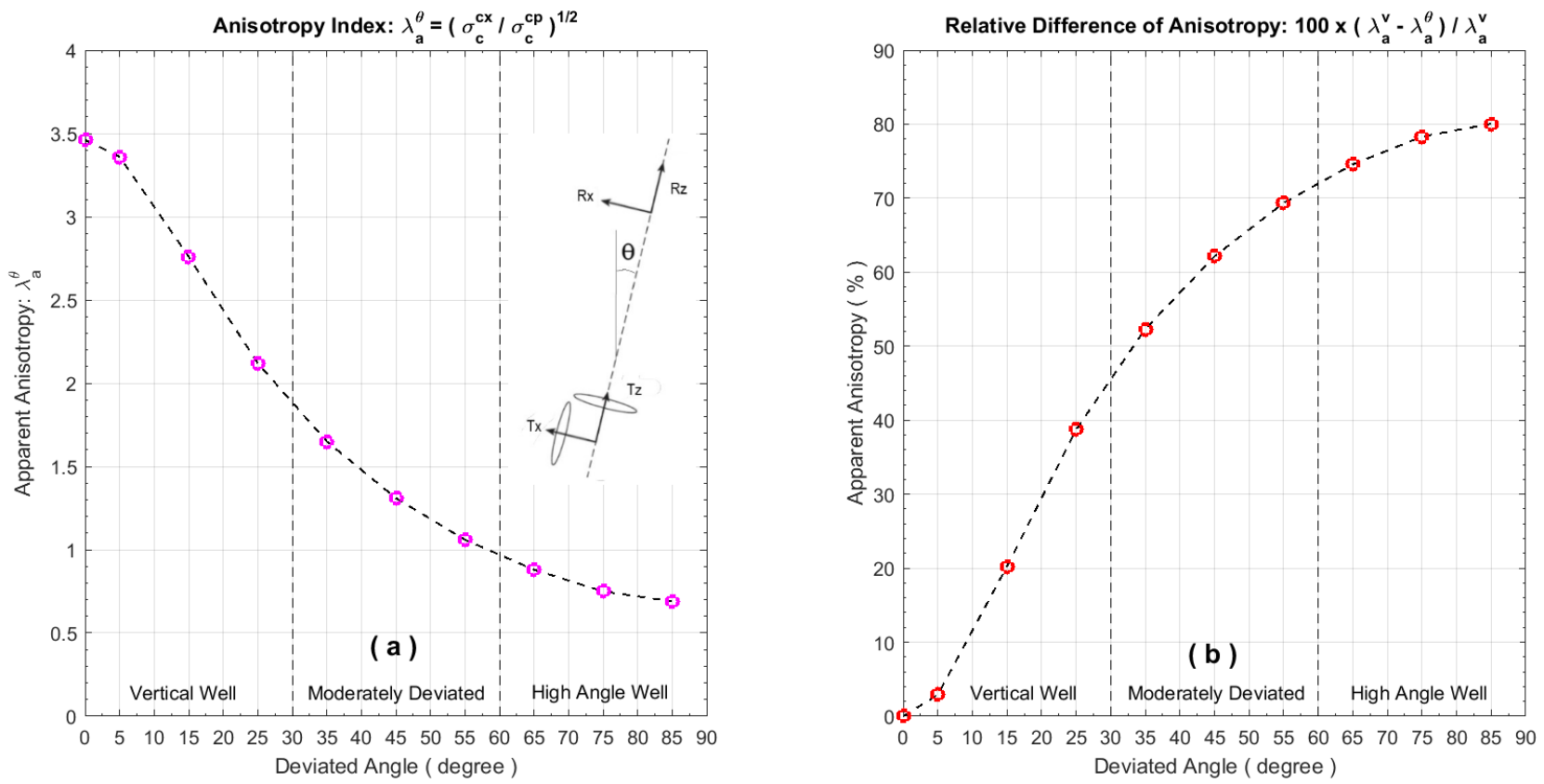

Figure 5 - Absolute (a) and relative (b) effect of the dip angle on the apparent anisotropy within the intrinsically anisotropic medium presented in Figure 4 at $z=-3 L$ below the interface.
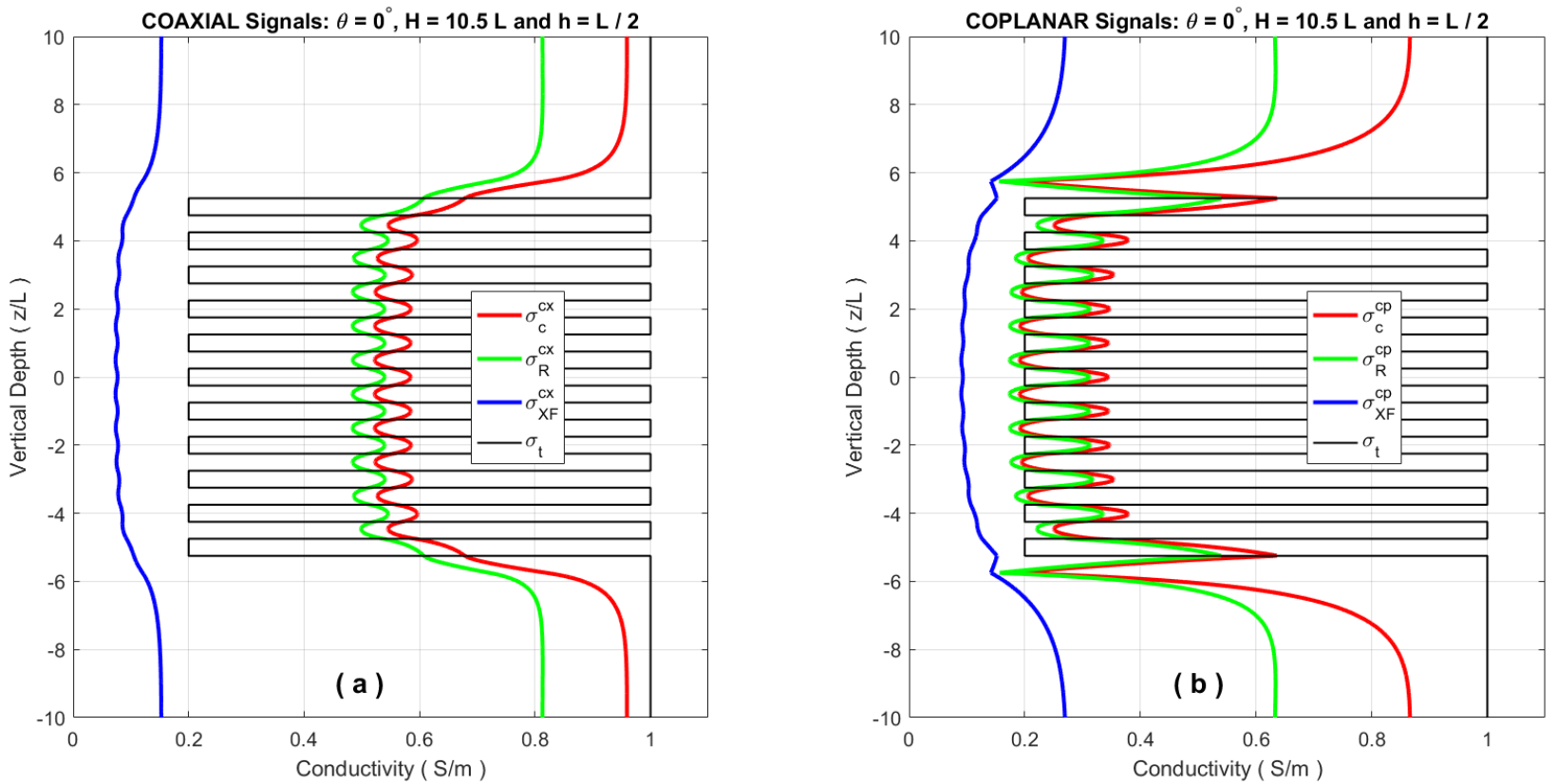

Figure 6 - Resistive, reactive and corrected vertical logs of the (a) coaxial and (b) coplanar coil arrays for a thick package $(H=10.5 L)$ of a laminated formation with symmetrical shoulders. 

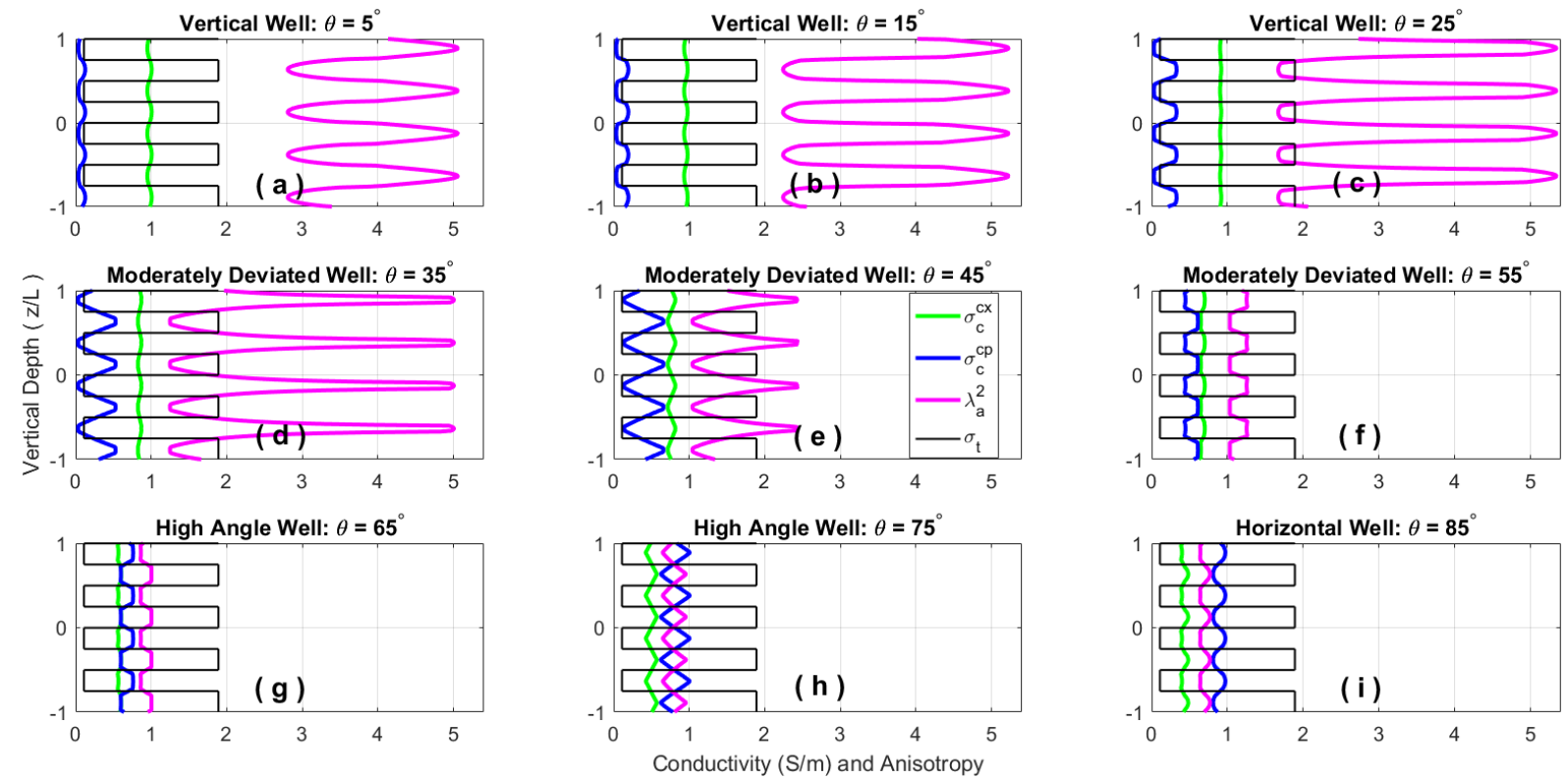

Figure 7 - Effect of the deviated angle on the anisotropy logs obtained by the coaxial and coplanar boosted signals at the center of a thinly $(h=L / 4)$ laminated formation.
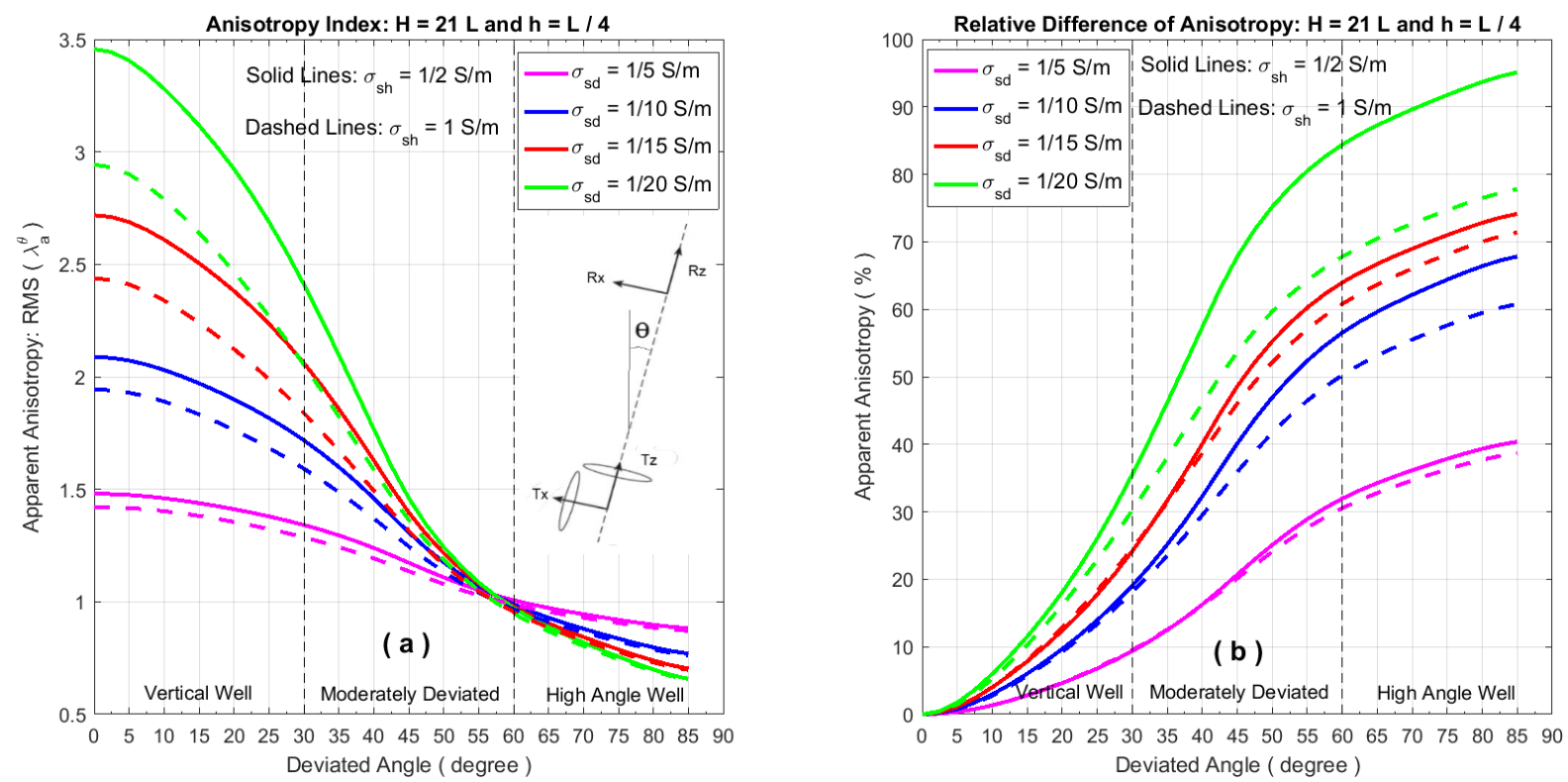

Figure 8 - Absolute (a) and relative difference (b) effect of the dip angle on the anisotropy logs at the center of a thick layer with structural anisotropy, as a thinly laminated sand-shale formation, with eight conductivity contrasts. 
signals show a smooth transition across the interface. We verify that this behavior is due to the lack of horizontal conductivity contrast between the two media, although it seems that the vertical electric field is still discontinuous. According to Anderson et al. (1992) these "horns" may be good quality interface indicators. Carvalho et al. (2010) show that as the dip angle increases the coplanar polarization horns are reduced progressively, and the logs tend to become straight lines at the horizontal limit.

Figure 7 shows the angle effect of deviated wells on the coaxial and coplanar corrected logs and in their resulting anisotropy logs. However, in this case, we consider: 1) the package thickness $(H=21 L)$ is twice the value of the previous example (Fig. 6); 2) the logs are at the center of the thick package, at depths ranging from $-2 L$ to $2 L$, that is, well away from the shoulder effects; and 3) the laminae thicknesses are half $(h=$ $L / 4$ ) of the previous case. The objective here is to simulate answers analogous to those shown in Figures 3 and 4, where the bottom bed is an intrinsic anisotropic medium with horizontal and vertical conductivities $\sigma_{h}=1.0 \mathrm{~S} / \mathrm{m}$ and $\sigma_{v}=0.2 \mathrm{~S} / \mathrm{m}$. Thus, through Eqs. (9) and (10) we calculate the equivalent sand-shale laminae conductivities: $\sigma_{s d}=0.11 \mathrm{~S} / \mathrm{m}$ and $\sigma_{s h}=1.89 \mathrm{~S} / \mathrm{m}$, respectively.

As the dip angle increases, the coaxial and coplanar logs show alternating features (smooth and angular) within the laminated formation, analogous to what is shown for vertical logs in Carvalho \& Régis (2016). These features change due to geometric and, mainly, polarization effects due to the discontinuous electric field at the interfaces. These effects determine not only the shape (smooth to angular) but also the magnitude of the oscillations, and they cause a curve reversal in some cases, such as at $65^{\circ}$. Anderson et al. (1992) show similar features in the coaxial logs at high values of conductivity contrasts (20 times), frequency ( $\mathrm{MHz}$ ) and dip angles $\left(60^{\circ}\right)$.

As the dip angle increases, the anisotropy logs undergo similar feature changes to those in the coaxial and coplanar corrected logs. The mean values of the anisotropy index decrease progressively, so that between $55^{\circ}$ and $65^{\circ}$ it is close to one (isotropic medium), just as it occurred in the intrinsically anisotropic medium observed in Figure 4.

Figure 8 shows the absolute (a) and relative (b) effect of the dip angle on the Root Mean Square (RMS) of the apparent anisotropy logs, at the center of a thick laminae package, with the same geometrical parameters of the previous case (Fig. 7), but now with eight different conductivity contrasts (from 5 to 40 times) between the sand-shale laminae.
The results in this case are analogous to those seen for the intrinsically anisotropic medium analyzed in Figure 5 , as expected. As the deviation angle ranges from $0^{\circ}$ to $85^{\circ}$, the anisotropy signal $\lambda_{a}^{\theta}$ decreases, starting from the value of $\lambda^{v}$, for the eight conductivity contrasts. These decays are more evident at the highest contrast, so that from $0^{\circ}$ to $30^{\circ}$ the apparent anisotropy drops approximately $9.5 \%$ for the lowest contrast (5 times) and $35.5 \%$ for the highest contrast (40 times), although these well deviations are still considered technically vertical.

\section{CONCLUSION}

This paper presents a quantitative analysis of the effects of the deviated angle in the apparent anisotropy logs, furnished by the tensor (triaxial) induction tools within one-dimensional (1D) laminated reservoir models and their equivalent anisotropic beds, in which the presence of the borehole and the invasion zones are neglected.

The results for both the laminated and its equivalent anisotropic model indicate that the coaxial and coplanar logs are very sensitive to the dip angle and, consequently, so is their resulting apparent anisotropy logs. As the dip angle ranges from $0^{\circ}$ to $30^{\circ}$ the apparent anisotropy drops approximately $9.5 \%$ for the lowest (5 times) conductivity contrast, and $35.5 \%$ for the highest (40 times) conductivity contrast, even though these well deviations are still considered technically vertical.

Although the technical literature recommends that when the anisotropy index is higher than five is an alert for the log analyst to look for potential laminated pay-reservoir, we verified that it is imperative to monitor the angle effects, since it strongly reduces this apparent anisotropy value furnished by these sets of sources and sensors, even in technically vertical wells. Otherwise, potential finely laminated reservoirs can be underestimated or even ignored.

\section{ACKNOWLEDGMENTS}

Paulo Carvalho thanks the Cyberspace Institute (ICIBE) of the Universidade Federal Rural da Amazônia (UFRA) for their support to this research.

\section{REFERENCES}

ANDERSON B, BARBER T, BASTIA R, CLAVAUD JB, COFFIN B, DAS M, HAYDEN R, KLIMENTOS T, MINH CC \& WILLIAMS S. 2008. Triaxial induction - A new angle for an old measurement. Oilfield Review, 20(2): 64-84. 
ANDERSON B, BONNER S, LULING MG \& ROSTHAL R. 1992. Response of 2-MHz LWD Resistivity and Wireline Induction Tools in Dipping Beds and Laminated Formations. The Log Analyst, 33(05). 34 pp.

CARVALHO PR \& RÉGIS CRT. 2016. Revisiting some old unexplained effects in induction logs within laminated formations with the triaxial induction tools. Brazilian Journal of Geophysics, 34(3): 393-404. doi: 10.22564/rbgf.v34i3.850.

CARVALHO PR \& VERMA OP. 1999. Coplanar coils response in a borehole. In: 6th International Congress of the Brazilian Geophysical Society. Rio de Janeiro, RJ, Brazil: SBGt.

CARVALHO PR, SANTOS WG, \& RÉGIS CRT. 2010. Fundamentals of coaxial and coplanar coil arrays in induction tools. Brazilian Journal of Geophysics, 28(1): 19-36. doi: 10.1590/S0102-261X2010000100002.

CLAVAUD JB, NELSON R \& GURU UK. 2005. Field Example of Enhanced Hydrocarbon Estimation in Thinly Laminated Formation with a Triaxial Array Induction Tool: A Laminated Sand-Shale Analysis with Anisotropic Shale. In: SPWLA 46th Annual Logging Symposium. Louisiana: SPWLA.

ELLIS DV \& SINGER JM. 2007. Well Logging for Earth Scientists. 2nd ed., Netherlands: Springer. doi: 10.1007/978-1-4020-4602-5.

KAUFMAN AA \& ITSKOVICH G. 2017. Basic Principles of induction logging - Electromagnetic Methods in Borehole Geophysics. Amsterdam: Elsevier. 520 pp.

KRIEGSHAUSER B, FANINI O, FORGANG S, ITSKOVICHG, RABINOVICH M, TABAROVSKY L, YU L, EPOV M, GUPTA P \& HORST JVD. 2000.
A New Multicomponent Induction Logging Tool to resolve Anisotropic Formations. In: SPWLA 41st Annual Logging Symposium. Texas: SPWLA.

MORAN JH \& KUNZ KS. 1962. Basic theory of induction logging and application to study of two-coil sondes. Geophysics, 27(6): 829-858. doi: 10.1190/1.1439108.

OMERAGIC D, BAYRAKTAR Z, THIEL M, HABASHY T, WU P, SHRAY $F$ \& ANTEZANA VHG. 2015. Triaxial Induction Interpretation in Horizontal Wells: Mapping Boundaries, and Characterizing Anisotropy and Fractures. In: SPWLA 56th Annual Logging Symposium. California: SPWLA.

PASSEY QR, YIN H, RENDEIRO CM \& FITZ DE. 2005. Overview of High-Angle and Horizontal Well Formation Evaluation: Issues, Learnings, and Future Directions. In: SPWLA 46th Annual Logging Symposium. Louisiana: SPWLA.

ZHANG Z, YU B \& LIU C. 2012. Investigation of Effects of Large Dielectric Constants on Triaxial Induction Logs. Applied Mathematics, 3(11A): 1811-1817. doi: 10.4236/am.2012.331246.

ZHDANOV MS, KENNEDY WD, CHERYAUKA AB \& PEKSEN E. 2001. Principles of Tensor Induction Well Logging in a Deviated Well in an Anisotropic Medium. In: SPWLA 42nd Annual Logging Symposium. Texas: SPWLA. 